\title{
La catastrophe de Biescas du 7 août 1996 ; analyse de la crue torrentielle du rio Aras dans les Pyrénées aragonaises (Espagne)
}

\author{
The Biescas disaster, August 7th 1996 ; geomorphic and hydraulic \\ reconstruction of the rio Aras flood (Aragon, Spain)
}

\author{
par Christian Lajournade $\left({ }^{1}\right)$, Christian Beaufrère $\left({ }^{1}\right)$, Gérard Lalanne-Berdouticq $\left({ }^{1}\right)$, \\ Francis Martignac ${ }^{(2)}$
}

(') Géologie Découverte Services (G.E.O.D.E.S.),

(2) Compagnie d'Aménagement des Côteaux de Gascogne

In the evening of August, 7th, 1996, a flash flood on the rio Aras near the Biescas village, killed eighty seven people, in a camping-site built on the alluvial fan. Geomorphic analysis shows a common dynamic at the peak flood in the three main torrents of the Aras catchment $\left(18,8 \mathrm{~km}^{2}\right)$, with successive and repetitive phase of erosion, sediment motion with mass flow and deposition. In this context, the water discharge reconstruction was difficult to establish. The numerous dams built against the erosion and solid motion in the final part of rio Aras could have been excellent supports to apply hydraulic formulas. But the catastrophic character of the flood, in a geodynamic sense, with broken dams and large, successive debris flows prohibits this approach. Nevertheless, we have reconstructed several flow sections on the three main tributaries, in the upper part of the catchment in order to estimate their specific water discharge. On these sections, the low sediment transport allows to calculate the discharge by the classical Manning-Strickler formula. The critical regime (Froude number $=1$ ) is a good approximation of the flow regime on steep slope channels [2-3]. We calculated the discharge taking into account the water depth as the critical one. This second approach allows to adjust the $K$ value for the Manning-Strickler equation. Then, we compute the total discharge for the whole catchment. Finally, we analysed the conditions in which the dams broke and the final grain flow which deposited about $70,000 \mathrm{~m}^{3}$ on the alluvian fan. Beyond the human drama, the rainfall intensity, $160 \mathrm{~mm} / 2 \mathrm{~h}$, the peak discharge, $180-210 \mathrm{~m}^{3} / \mathrm{s}$ which is about twice more than the 10,000 years flood discharge, show the extreme and exceptional character of this event which is a reference for the probable maximum torrential flood (PMF) in the occidental area of the Pyrenees.

\section{IINTRODUCTION}

Le 7 août 1996, une pluie orageuse de forte intensité s'est abattue sur la région de Biescas, provoquant une crue violente sur le rio Aras, affluent du rio Gallego, une des principales rivières des Pyrénées aragonaises. L'inondation brutale du camping implanté sur le cône de déjection du rio Aras a provoqué la mort de 87 personnes. L'ampleur de cette catastrophe humaine et l'aménagement exceptionnel du bassin versant pour contenir l'activité torrentielle, confèrent à cet événement dramatique et douloureux, un intérêt de premier ordre en matière d'hydrologie extrême, de transports solides et de prévention des risques torrentiels.

La reconstitution d'un tel événement, entreprise sitôt après la catastrophe, s'est révélée difficile. Nous avons conduit de minutieuses investigations sur les affluents et le cours terminal de l'Aras pour approcher une réalité qui, à bien des égards, restera opaque du fait de l'importance des bouleversements géomorphologiques et de la parcimonie des données pluviométriques. Il nous paraissait cependant fondamental d'estimer les débits liquides qui seuls permettent d'évaluer et de relativiser l'intensité d'un tel phénomène.

Après une analyse du phénomène pluvieux générateur de la crue, nous présentons ces aspects géomorphologiques et 


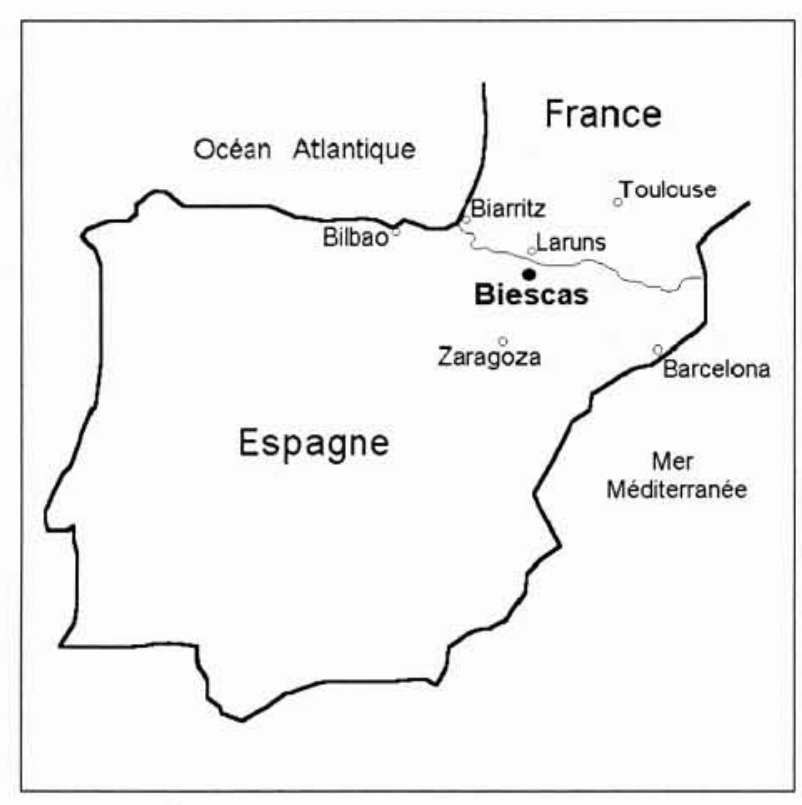

1. Cadre géographique. notamment la dynamique des transports solides, puis l'analyse des débits liquides dans les trois bassins versants alimentant le rio Aras. Deux volets de la dynamique de la crue sur le rio Aras sont ensuite abordés : le comportement des ouvrages de correction torrentielle et les modalités du transport solide. Pour conclure, nous tirons les enseignements généraux et régionaux de cet événement majeur.

\section{II — LE BASSIN VERSANT DU RIO ARAS}

Le bassin versant couvre une superficie de $18,8 \mathrm{~km}^{2}$ et présente une morphologie typique de bassin torrentiel. On distingue trois sous-bassins principaux, le rio Aso $\left(10,5 \mathrm{~km}^{2}\right)$, le rio Betes $\left(4,38 \mathrm{~km}^{2}\right)$ et le rio Selva $\left(2,75 \mathrm{~km}^{2}\right)$. Ils confluent au même point pour former le rio Aras (fig. 2).

Le paysage est marqué par l'importante couverture boisée d'affinité méditerranéenne, dominée par la pinède et la chênaie. La limite supérieure de la forêt se situe vers $1700 \mathrm{~m}$ d'altitude. Les versants supraforestiers correspondent à des landes herbacées utilisées pour l'élevage extensif. Malgré la vigueur des pentes, aucune zone d'érosion vive n'affecte le flysch gréso-pélitique éocène qui constitue le substrat rocheux.

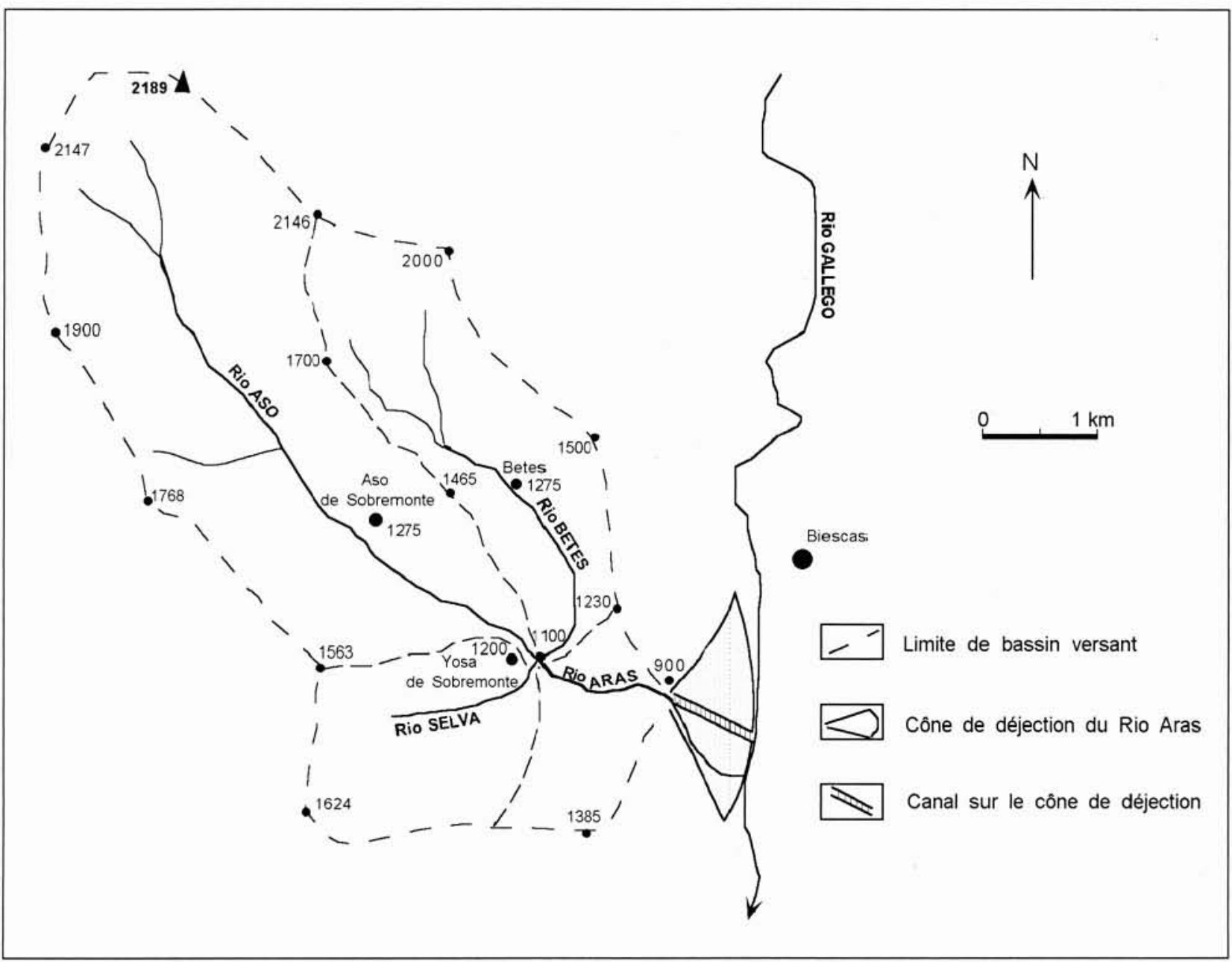

2. Le bassin du rio Aras. 
Dans leur cours terminal, ces torrents s'encaissent dans un puissant complexe de matériaux détritiques (argiles lacustres, dépôts torrentiels, moraines) très sensibles à l'érosion. De même après la confluence, le Rio Aras s'engorge profondément dans les dépôts morainiques et débouche sur un puissant cône de déjection de 52 ha, traversé par la route nationale 136. Le camping "Las Nieves » était installé sur la partie méridionale de ce cône.

\section{ANALYSE DE LA PLUIE GÉNÉRATRICE}

\subsection{Intensité-durée-fréquence}

Les données pluviométriques ne permettent pas une analyse précise de la séquence pluvieuse sur le bassin versant. L'orage s'est produit entre $17 \mathrm{~h} 30$ et $19 \mathrm{~h} 30$. L'unique pluviomètre, installé à Aso de Sobremonte et d'une contenance de $100 \mathrm{~mm}$, a débordé durant l'épisode. Hors du bassin versant, le pluviomètre de Biescas a recueilli $160 \mathrm{~mm}$ soit une intensité moyenne de $80 \mathrm{~mm} / \mathrm{h}$. L'intensité maximale est supérieure car le paroxysme de la pluie se concentre sur la deuxième heure.

L'intensité et la durée de la pluie sont conformes aux valeurs des pluies extrêmes enregistrées sur les réseaux pluviométriques français et espagnols dans la région des Pyrénées occidentales depuis près de 50 ans [1].

Pour analyser la fréquence de ces pluies extrêmes, on ne dispose sur les postes montagnards que de séries pluviométriques au pas de $24 \mathrm{~h}$. Le tableau 1 présente les valeurs de pluie $/ 24 \mathrm{~h}$ selon différentes périodes de retour à Biescas et à Laruns situé en vallée d'Ossau sur le versant français.

Tableau 1. - Valeurs de la pluie/24 h pour différentes périodes de retour sur les stations de Biescas, Laruns et Biarritz. (max. annuel ; 1 valeur/an ; loi de Gumbel).

\begin{tabular}{|l|c|c|c|}
\hline $\begin{array}{l}\text { Période } \\
\text { de retour }\end{array}$ & $\begin{array}{c}\text { Biescas } \\
(1940-1996)\end{array}$ & $\begin{array}{c}\text { Laruns } \\
(1946-1996)\end{array}$ & $\begin{array}{c}\text { Biarritz } \\
(1961-1992)\end{array}$ \\
\hline 100 ans & $156 \mathrm{~mm}$ & $176 \mathrm{~mm}$ & $169 \mathrm{~mm}$ \\
\hline 250 ans & $176 \mathrm{~mm}$ & $196 \mathrm{~mm}$ & $193 \mathrm{~mm}$ \\
\hline 500 ans & $192 \mathrm{~mm}$ & $212 \mathrm{~mm}$ & $211 \mathrm{~mm}$ \\
\hline 1000 ans & $208 \mathrm{~mm}$ & $228 \mathrm{~mm}$ & $229 \mathrm{~mm}$ \\
\hline Gradex & $22,3 \mathrm{~mm}$ & $22,3 \mathrm{~mm}$ & $26 \mathrm{~mm}$ \\
\hline
\end{tabular}

Régionalement, sur le réseau Météo-France, les valeurs de la pluie de $24 \mathrm{~h}$ de la station de Biarritz sont analogues à celles des postes de Biescas et de Laruns (tabl. 1). L'extrapolation des valeurs d'intensité-durée-fréquence de Biarritz, ajustées sur la loi de Gumbel, indique une valeur de $96 \mathrm{~mm}$ pour la pluie de 2 heures de fréquence $1 / 10000$, soit très en deçà du cumul de $160 \mathrm{~mm} / 2 \mathrm{~h}$ enregistré à Biescas. Il est donc vain de situer la périodicité de tels phénomènes par l'approche statistique en l'état actuel des données pluviométriques.

\subsection{Contexte météorologique}

La situation du 7 août 1996 est typique des périodes orageuses estivales dans les Pyrénées occidentales, qu'il s'agisse du versant nord ou du versant sud de la chaîne. Dans un champ de pression à faible gradient (marais baro- métrique), un front froid est associé à une "goutte froide " d'altitude $\left(-12{ }^{\circ} \mathrm{C}\right.$ à $\left.5500 \mathrm{~m}\right)$. Les températures diurnes étant supérieures à $30^{\circ} \mathrm{C}$ au sol, le gradient thermique vertical est de l'ordre de $0,75^{\circ} \mathrm{C} / 100 \mathrm{~m}$ favorisant la formation de cellules orageuses très actives, les effets orographiques accentuant la dynamique thermo-convective.

\section{D DYNAMIQUE DE LA CRUE DANS LES AFFLUENTS}

\subsection{Analyse morphosédimentaire}

A la pointe de la crue, le transport solide détermine les modalités d'écoulement. Il se propage selon un processus de substitution de la charge sédimentaire intégrant trois phases distinctes.

- Une phase 1 d'érosion du lit et des berges qui s'effectue bien au-delà du dépavage et détermine un recalibrage variable en fonction de la pente, de la nature et de l'épaisseur des formations superficielles encaissantes.

- Une phase 2 à fort transport solide, généralement par écoulement en masse ${ }^{1}$ qui n'est pas érosif sur les zones à pente égale ou proche de la pente limite d'écoulement (5$6 \%$ ) du fait de vitesses faibles. Sur les sections à plus forte pente (>8-10\%), l'écoulement en masse est fortement érosif.

- Une phase 3 de sédimentation qui s'effectue :

- par arrêt "naturel " de la coulée sur des pentes de 4-5\% ou par blocage sur un amas de blocs de gros volumes,

- par blocage «artificiel " au niveau d'un ouvrage formant barrage (pont, barrage non comblé), d'une piste recoupant un talweg ou d'un bief contrôlé par un seuil de correction torrentielle.

La reprise érosive à l'aval de la zone de sédimentation est toujours brutale.

La cartographie des principales zones de dépôts associées à des coulées de débris $(\mathrm{C} 1$ à $\mathrm{C} 12$, fig. 3$)$ montrent que ce schéma de fonctionnement de la crue est valide sur l'intégralité du cours du rio Betes et du rio Selva. Sur le rio Aso, il ne s'applique que dans la partie terminale du torrent sur un linéaire de $1 \mathrm{~km}$ environ.

\subsection{Rio Betes}

Sur les talwegs supérieurs, qui drainent des surfaces de moins d'un $\mathrm{km}^{2}$, la géomorphologie montre que la crue est déjà intense. Ces talwegs élémentaires ont été parcourus par des écoulements très érosifs contrôlés par des ruptures d'embâcles (photo $\mathrm{n}^{\circ} 1$ ). Sur le rio Betes, les principaux corps sédimentaires sont associés à des coulées de débris. Les coulées $\mathrm{C} 3$ et $\mathrm{C} 5$ se sont bloquées sur les ponts routiers amont et aval respectivement. La coulée $\mathrm{C} 4$ a sédimenté sur une pente de $4 \%$ dans un élargissement du chenal. A l'aval, la reprise érosive (phase 1) est extrêmement rapide et spectaculaire du fait du creusement d'un néo-chenal (photo $\mathrm{n}^{\circ} 2$ ).

A l'aval de la coulée $\mathrm{C} 7$, le rio Betes s'engorge dans un bief à très forte pente, $20 \%$ en moyenne. La reprise érosive très brutale en tête du bief puis l'écoulement en masse sur cette forte pente ont déterminé une sorte d'emballement dynamique mobilisant les méga-débris contenus dans les moraines et recalibrant profondément le chenal. Des glisse-

(1) On emploira indifféremment par la suite les termes d'écoulement en masse, de coulée de débris et de lave torrentielle. 
ments de versants ont directement alimenté la coulée de débris. Les deux seuils construits dans le bief terminal ont été totalement emportés. A l'exutoire du torrent, la confluence avec le rio Aso est couverte par un amoncellement chaotique de 3 à $4 \mathrm{~m}$ d'épaisseur (dépôts de la coulée C8, photo $\mathrm{n}^{\circ} 3$ ). Tous les débris portent des marques d'impact qui témoignent de l'importance des interactions granulaires.

\subsection{Rio Aso}

La géomorphologie met en évidence une intumescence brutale de la crue dans la partie terminale du rio. Elle s'explique par la rupture d'un embâcle dans le cours terminal d'un petit affluent qui draine le versant au nord du village d'Aso de Sobremonte (fig. 3). La débâcle a littéralement défoncé le chenal primitif et généré un transport solide en charriage démesuré à l'échelle de ce bassin versant élémentaire.

A l'aval de cette confluence, le lit de l'Aso qui ne présente pas vers l'amont de marques d'érosion, apparaît de plus en plus «défoncé » au fur et à mesure de son encaissement dans les formations glaciaires. Il est probable qu'une lave torrentielle se soit rapidement constituée (phase 2), évoluant sur une pente de 8 à $10 \%$ jusqu'au pont de Yosa qui a été mis en charge (dépôts sur la chaussée) avant de céder ${ }^{2}{ }^{2}$ ).

L'écoulement dynamisé par la rupture du pont a de nouveau fortement érodé les berges et provoqué des glissements dans les moraines sur un linéaire d'environ $250 \mathrm{~m}$. L'abaissement de la pente (4-5\%) imposé par un seuil de correction torrentielle, a provoqué la sédimentation de la coulée de débris. Son volume est d'environ $5000 \mathrm{~m}^{3}$.

A l'aval du seuil, l'écoulement liquide a affouillé le pied de l'ouvrage mais sans altérer sa stabilité et franchit l'ultime seuil situé à moins de $50 \mathrm{~m}$. Au-delà, la crue liquide n'a que légèrement érodé le lit: les seuils successifs ont ainsi eu pour effet de limiter la reprise d'érosion, en cassant le profil en long à l'exutoire de l'Aso. Celui-ci apparaît donc peu marqué par la crue.

\subsection{Rio Selva}

Nous avons relevé trois zones de dépôts principales. La zone de dépôt supérieure $(\mathrm{C} 10)$ correspond à un corps de coulée de débris d'environ $1000 \mathrm{~m}^{3}$ immobilisé au niveau d'une piste recoupant le talweg, imposant donc une diminution brutale de la pente.

Vers l'aval, la géomorphologie indique que l'intensité de la crue est ici comparable à celle enregistrée sur le cours terminal du rio Aso. Le comportement des deux seuils construits sur le cours terminal corrobore cette analyse. Ils ont, comme sur l'Aso, parfaitement contenu les flux solides (C11 et $\mathrm{C} 12)$ sans subir de dommages importants. L'ouvrage amont, probablement le plus récent de tous car construit en béton, s'est cependant affaissé en rive droite par affouillement dans les argiles lacustres.

\section{V — RECONSTITUTION DES DÉBITS LIQUIDES}

En l'absence de données sur la séquence pluvieuse, quant à sa chronologie précise et sa spatialisation, les débits ne peuvent être reconstitués que par une approche hydraulique.

(2) La rupture du pont et la débâcle ont été présentées comme la cause de la catastrophe au niveau du camping. En fait, l'impact de cette débâcle est tout à fait négligeable, du fait de la distance (environ $2 \mathrm{~km}$ ) et du volume de la retenue, de l'ordre de $3000 \mathrm{~m}^{3}$, qui contenait au moins autant de sédiments que d'eau.

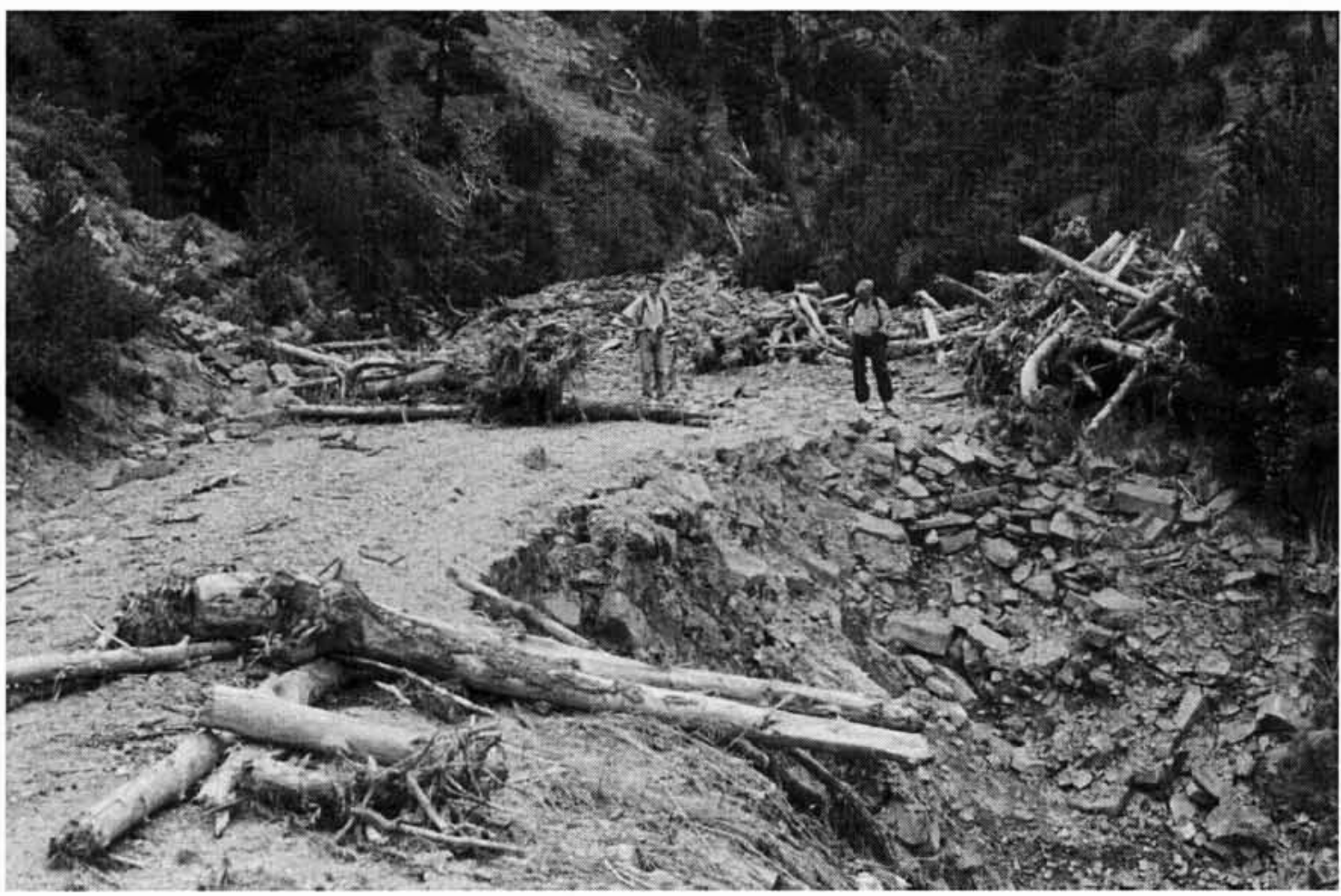

Photo $\mathrm{n}^{\circ}$ 1. Coulée de débris $\mathrm{C} 1$; noter le volume important d'arbres et l'importance de la reprise érosive (phase 1) affectant la piste. 


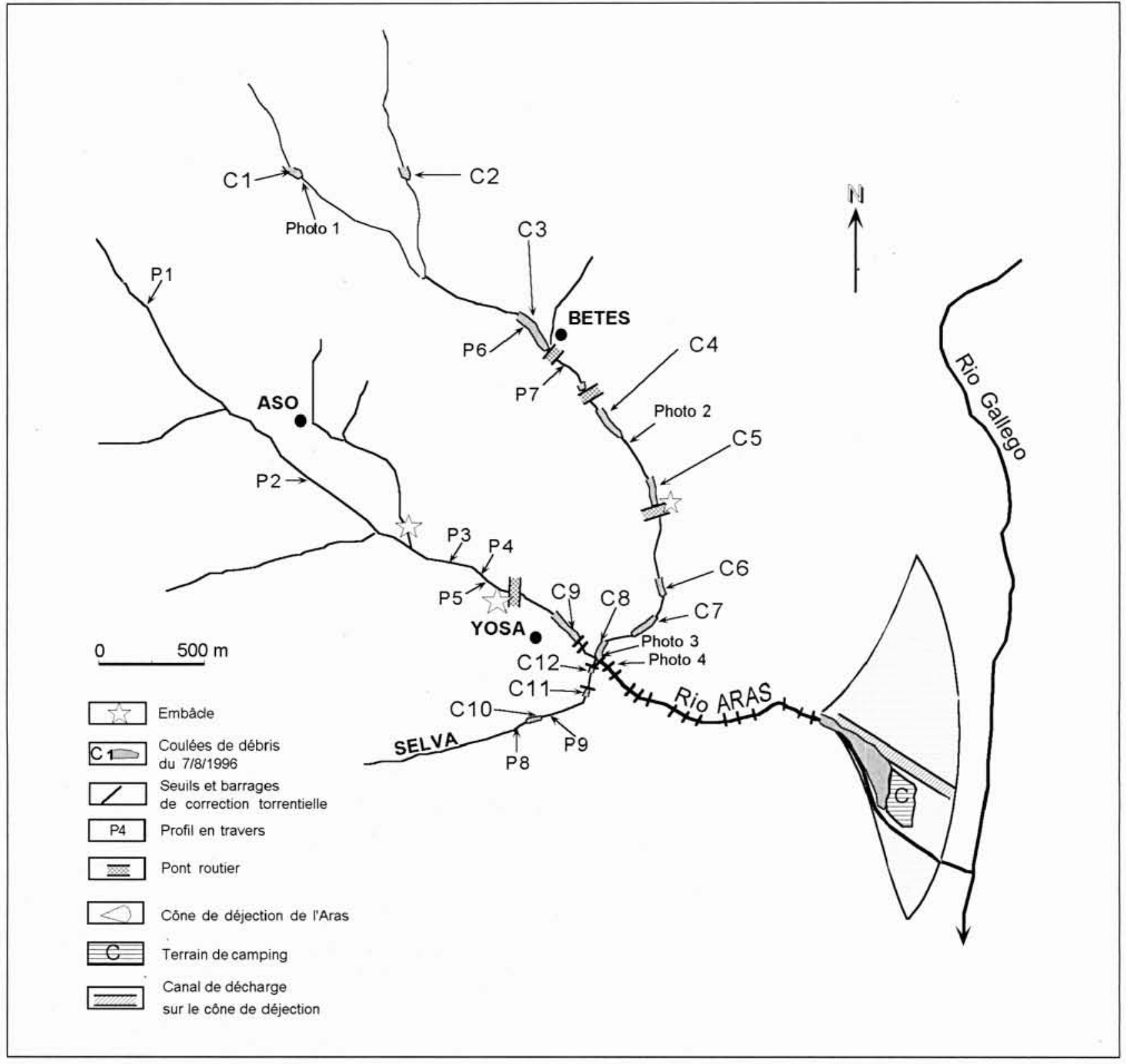

3. Eléments de l'analyse dynamique de la crue.

Cependant, la dynamique de la crue s'est caractérisée par un transport solide important et par un recalibrage parfois considérable des lits. Pour de tels écoulements, la reconstitution des débits liquides est délicate. Aussi, l'objectif principal est de situer des ordres de grandeur « réalistes".

L'importance des désordres morphologiques, l'indétermination des conditions d'écoulement, la forte végétalisation des plages de dépôts en amont des ouvrages et la valeur même du débit sont autant d'éléments défavorables à une analyse fiable des débits liquides dans le chenal de l'Aras. Nous avons donc cherché à estimer les débits spécifiques sur les trois affluents. Sur la base de l'analyse géomorphologique, nous avons recherché des sites caractérisés par un transport solide en charriage faible, donc situés à l'aval immédiat d'une zone de dépôts (phase 3 ). De plus, nous avons recherché les sections de contrôle sur les ouvrages non-endommagés.

L'équation classique de Manning-Strickler apparaît inadaptée pour un fort transport solide en charriage [2]. Cependant, nous l'avons utilisée car les sections d'écoulement retenues comme référence présentaient un transport solide faible à la pointe de la crue. Chaque fois que possible, nous avons confronté cette approche à un calcul basé sur la hauteur critique. En effet sur forte pente, le nombre de Froude oscillant rapidement de part et d'autre de l'unité, le régime critique peut constituer une approximation acceptable des conditions d'écoulement [2-3]. Le calcul sur la hauteur critique a conduit à confirmer ou à fixer rétrospectivement la valeur du coefficient de rugosité $K$ de la formule de Manning-Strickler. 


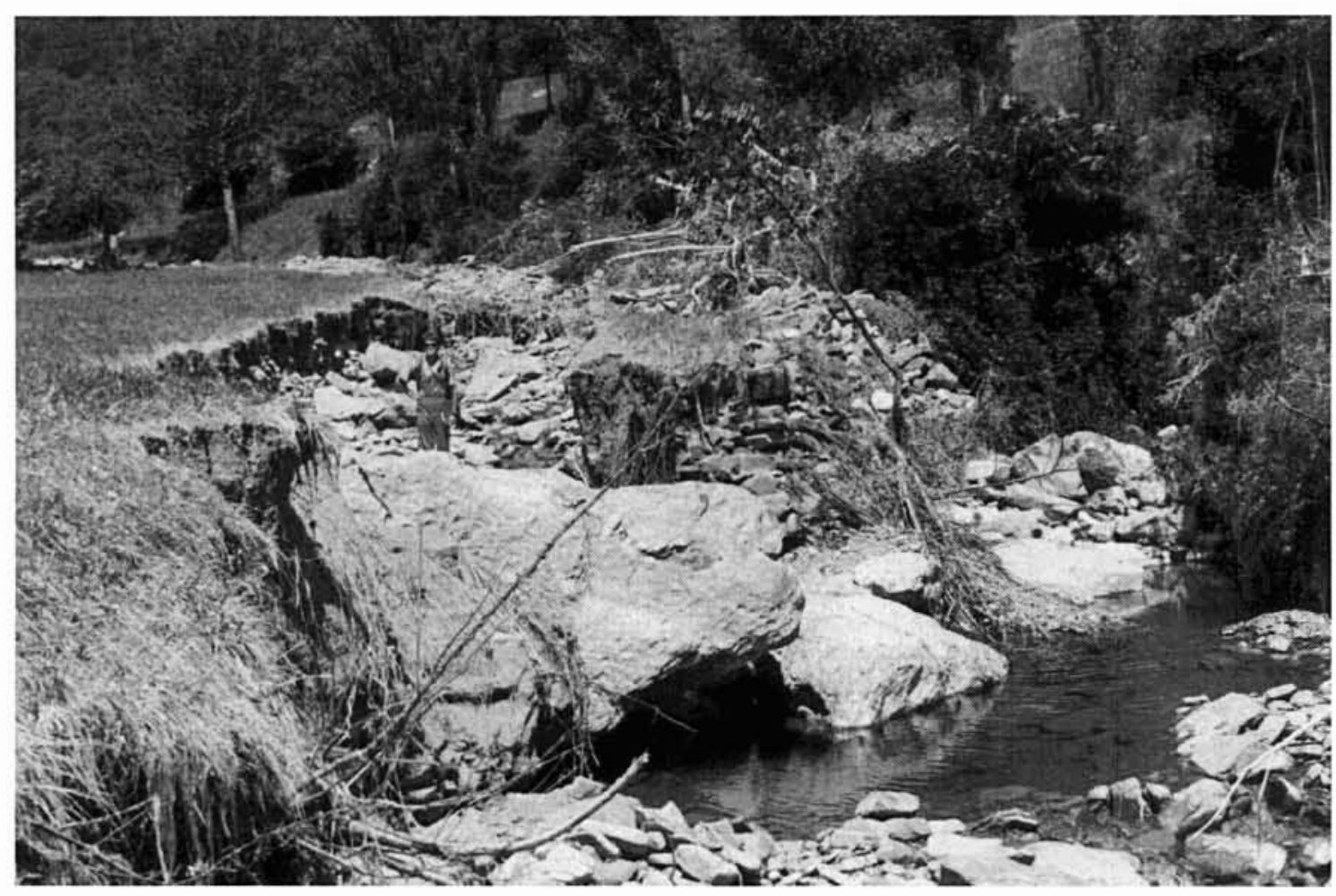

Photo $\mathrm{n}^{\circ}$ 2. Chenal du rio Betes à $50 \mathrm{~m}$ à l'aval de la coulée de débris $\mathrm{C4}$, au second plan ; noter l'importance de la reprise érosive.

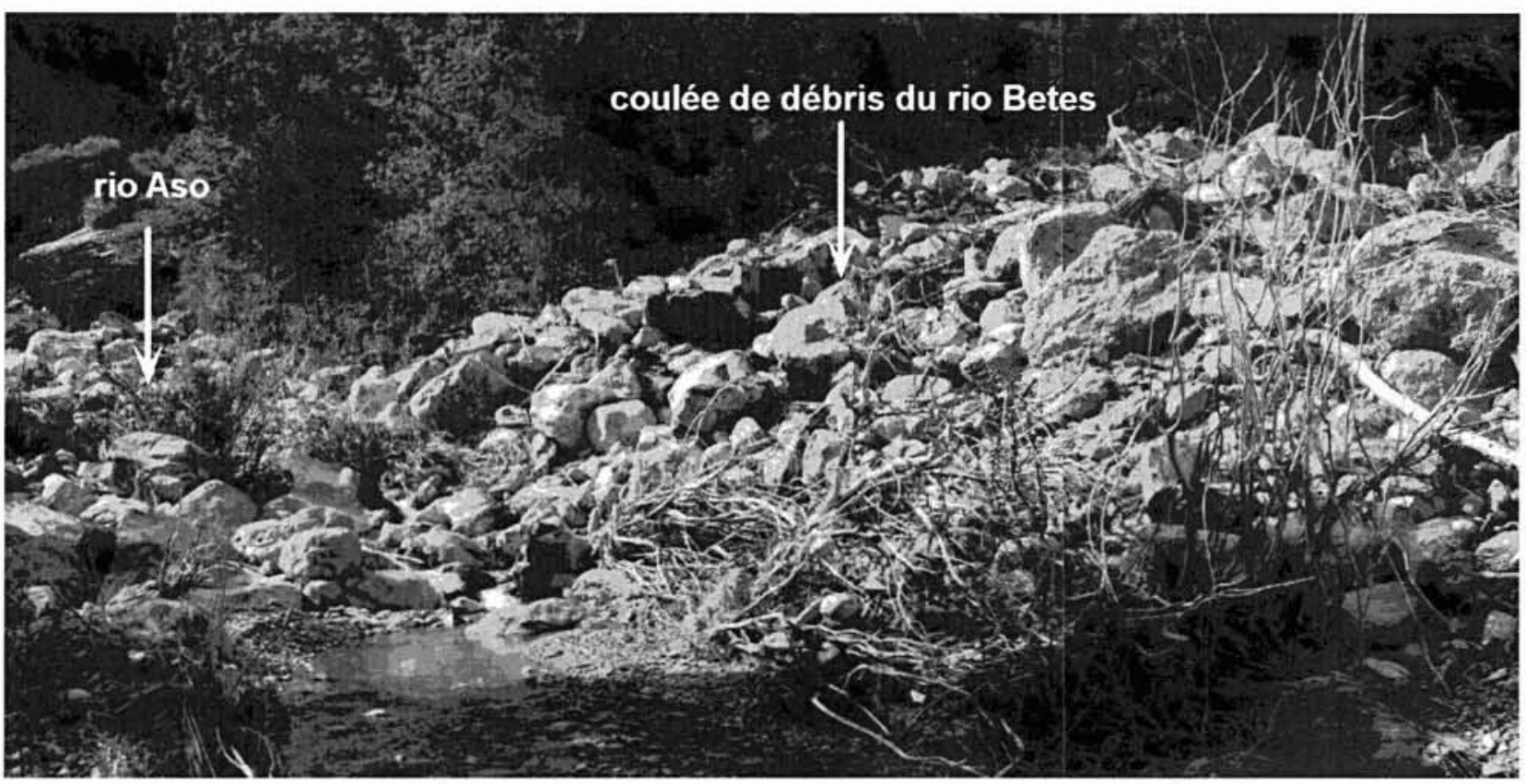

Photo $n^{\circ}$ 3. Confluence Aso-Betes, coulée de débris C8

- 5.1 Estimation des débits de pointe au niveau des différents profils

Nous présentons les résultats obtenus sur 9 profils en travers répartis sur les 3 torrents (fig. 2). Ces profils sont d'une "qualité » hydraulique inégale, mais il était intéressant de montrer les difficultés d'une reconstitution des débits pour ce type de crue. Pour chaque cours d'eau, nous avons retenu un site unique faisant référence pour l'estimation du débit spécifique de pointe. Ces sites présentent la «meilleure » fiabilité : remaniement négligeable de la géométrie initiale du lit, section de contrôle au niveau d'un ouvrage, faible transport solide.

Le tableau 2 présente toutes les caractéristiques géométriques des profils servant aux calculs des débits. 
Tableau 2. - Données des calcul des débits liquides sur les profils caractéristiques.

\begin{tabular}{|c|c|c|c|c|c|c|c|c|c|c|c|}
\hline ASO & Alt./site & $\begin{array}{c}\text { Surf. } \\
\mathrm{km}^{2}\end{array}$ & $\begin{array}{c}\text { Section } \\
\mathrm{m}^{2}\end{array}$ & $\begin{array}{c}\text { L. } \\
\mathrm{m}\end{array}$ & $\begin{array}{c}H . \\
\mathrm{m}\end{array}$ & $\begin{array}{c}\mathrm{I} \\
\%\end{array}$ & $R$ & $K$ & $\begin{array}{c}\text { Qmax } \\
\mathrm{m}^{3} / \mathrm{s}\end{array}$ & $\begin{array}{c}V \mathrm{moy} \\
\mathrm{m} / \mathrm{s}\end{array}$ & $\begin{array}{c}q \mathrm{max} \\
\mathrm{m}^{3} / \mathrm{s} / \mathrm{km}^{2}\end{array}$ \\
\hline$P 1$ & $1300 \mathrm{~m}$ & 6,8 & 7,85 & 9,1 & 1,35 & 13 & 0,8 & 10 & 25 & 3,2 & 3,7 \\
\hline$P 2$ & $1240 \mathrm{~m} /$ seuil & 8,5 & 8,70 & 10 & 0,90 & 5,5 & 0,71 & 20 & $27 / 32 \rightarrow 30$ & $3 / 3,5$ & 3,5 \\
\hline BETES & & & & & & & & & & & \\
\hline$P 6$ & $300 \mathrm{~m}$ amont village & 2,8 & 14,5 & 9 & 1,60 & 6,7 & 1,2 & 15 & $57 / 63,5$ & $3,9 / 4,4$ & 21,4 \\
\hline$P 7$ & $150 \mathrm{~m}$ aval village & 3,2 & 16 & 10 & 1,60 & 6 & 1 & 15 & $63 / 59 \rightarrow 60$ & 3,7 & 18,7 \\
\hline SELVA & & & & & & & & & & & \\
\hline$P 8$ & $1150 \mathrm{~m}$ & 2 & 11,5 & 8,5 & 1,30 & 15 & 0,66 & 12 & $39,5 / 40,5 \rightarrow 40$ & $3,4 / 3,5$ & 20 \\
\hline$P 9$ & aval piste & 2,15 & 8,70 & 11 & 1,30 & 8 & 0,7 & 20 & 39 & 4,5 & 18,6 \\
\hline
\end{tabular}

\subsubsection{Rio Aso: $P_{1}$ à $P_{5}$}

Ces profils se répartissent entre $1300 \mathrm{~m}$ et $1170 \mathrm{~m}$ d'altitude, soit un linéaire d'environ $2 \mathrm{~km}$. Jusque vers $1220 \mathrm{~m}$ d'altitude s'ébauche une plaine alluviale construite par les divagations torrentielle, puis le torrent s'encaisse dans les formations würmiennes (argiles noires lacustres, alluvions torrentielles autochtones, moraines à gros blocs).

- $P_{1}$ : Il se situe à environ $1 \mathrm{~km}$ en amont du village d'Aso. Le chenal principal a contenu la crue, aucune trace d'écoulement n'étant visible dans les chenaux latéraux. Le pavage du lit, à gros galets et blocs, ne paraît pas avoir subi d'évolution importante malgré la pente de $13 \%$. Ces observations indiquent que la crue n'était pas extrême.

- $P_{2}$ : Il est situé sur le premier seuil de correction torrentielle qui a parfaitement entonné la crue et n'a pas été endommagé : il s'agit donc d'un site de première importance. Le calcul en hauteur critique confirme les observations faites sur P1 : la crue n'était pas extrême à ce point du bassin. Cela conduit à confirmer rétrospectivement pour P1 une valeur de $K=10$.

Les débits spécifiques obtenus sont analogues, 3,2 et $3,7 \mathrm{~m}^{3} / \mathrm{s} / \mathrm{km}^{2}$ pour 6,8 et $8,5 \mathrm{~km}^{2}$ respectivement sur $P_{1}$ et $P_{2}$. Les valeurs obtenues sur $P_{2}$ sont finalement retenues.

- $P_{3}-P_{4}-P_{5}$ : Ces profils se répartissent sur $400 \mathrm{~m}$ en amont du pont de Yosa. Ils se caractérisent par un accroissement spectaculaire de la section d'écoulement $\left(32,5-30,5-24 \mathrm{~m}^{2}\right.$ respectivement). Cette évolution témoigne bien de l'exacerbation de la crue induite notamment par les apports démesurés (régime de débâcle) du petit affluent rive gauche. Le lit encaissé dans les moraines à gros blocs a été profondément recalibré. Aussi, ces sections ne sont pas fiables.

\subsubsection{Rio Betes : $P_{6}-P_{7}$}

Ces profils se situent de part et d'autre du village de Betes.

A l'amont, la section $P_{6}$ correspond à une néo-incision dans le corps de la coulée de débris (C3) bloqué sur le pont du village. La qualité de la section est donc douteuse puisqu'il s'agit d'une zone d'érosion. A l'inverse, le profil $P_{7}$ situé à l'aval du pont de Betes, au début d'une phase 1 , présente l'intérêt d'avoir été faiblement retouchée par les écoulements liquides. Elle présente donc une bonne fiabilité.

Les profils, de forme rectangulaire, permettent les calculs selon les deux approches retenues. Les résultats sont analogues sur les deux sites. La station $P_{6}$ surestime effectivement les débits car, vers l'aval, les apports latéraux ayant été importants, on devrait obtenir une différence significative entre les 2 stations. Du fait de sa qualité, le résultat obtenu sur la station $P_{7}$ constitue une référence fiable. On obtient donc pour $3,2 \mathrm{~km}^{2}$ de bassin versant un débit spécifique de $18,7 \mathrm{~m}^{3} / \mathrm{s}^{2} \mathrm{~km}^{2}$

\subsection{3 $P_{8}$ et $P_{9}$ sur le Selva}

Ces profils se situent de part et d'autre d'une piste qui recoupe le lit du torrent. L'atterrissement de la piste a bloqué une coulée granulaire (phase 3 ) constituée à l'aval du profil $P_{8}$, situé sur une zone d'érosion du chenal (phase 1). $P_{8}$ surestime probablement la section noyée réelle, à l'instar de $P_{6}$.

A l'aval de la piste, l'écoulement liquide a franchi un ressaut de $4 \mathrm{~m}$ pour retrouver le chenal naturel. Le profil $P_{9}$, à une vingtaine de mètres à l'aval du ressaut, n'a pas subi d'érosion, tant en lit mineur que sur les berges (herbes simplement couchées). Le transport solide en charriage était donc très faible.

Le débit calculé à $P_{8}$ sur la hauteur critique conduit à fixer $K=12$. Cette section étant sur une zone d'érosion, le calcul surestime probablement le débit réel. Cette valeur par excès peut néanmoins servir à caler la valeur de $K$ sur $P_{9}$, compte tenu de la faible évolution de la superficie de l'impluvium. Donc pour $P_{9}$, on est conduit à adopter une valeur de 20 qui semble cohérente avec la rugosité du radier (absence de blocs) et la surface herbeuse des berges. On obtient ainsi pour $P_{8}$ et $P_{9}$ un débit de pointe de l'ordre de $40 \mathrm{~m}^{3} / \mathrm{s}$ pour un bassin versant de 2 à $2,15 \mathrm{~km}^{2}$, soit au niveau de $P_{9}$, un débit spécifique de $18,6 \mathrm{~m}^{3} / \mathrm{s} / \mathrm{km}^{2}$.

\subsection{Les débits liquides de pointe à l'exutoire du rio Aso}

Les débits spécifiques obtenus sur le Betes et sur le Selva sont analogues. A l'inverse, $80 \%$ du bassin de l'Aso n'a pas connu une forte crue. Vers l'exutoire, la géomorphologie témoigne bien d'un gonflement très brutal de la crue de l'Aso qui s'exprime par l'apparition d'un transport solide en masse associée à un recalibrage vigoureux du chenal : ces caractéristiques sont celles identifiées sur les deux autres bassins.

Aussi, peut-on proposer une estimation des débits à l'exutoire de l'Aso, sur la base des débits spécifiques calculés sur le Betes et sur le Selva, en considérant sur la partie terminale du bassin, soit $2 \mathrm{~km}^{2}$, un débit spécifique de l'ordre de $18,5 \mathrm{~m}^{3} / \mathrm{s} / \mathrm{km}^{2}$ (tabl. 3). En considérant que les pointes des hydrogrammes du bassin amont et de la zone aval sont soit tout à fait décalées soit parfaitement synchrones, on obtient un débit de pointe compris entre 37 et $67 \mathrm{~m}^{3} / \mathrm{s}$. Dans cette fourchette, les débits spécifiques sont donc entre 3,5 et $6 \mathrm{~m}^{3} / \mathrm{s} / \mathrm{km}^{2}$ pour les $10,5 \mathrm{~km}^{2}$ de bassin versant de l'Aso. 
Tableau 3. - Synthèse des calculs des débits liquides.

\begin{tabular}{|c|c|c|c|c|}
\hline & $\begin{array}{c}\text { qmax. } \\
\text { de référence } \\
\text { en } \mathrm{m}^{3} / \mathrm{s} / \mathrm{km}^{2}\end{array}$ & $\begin{array}{c}\text { Qmax. } \\
\text { de référence } \\
\text { en } \mathrm{m}^{3} / \mathrm{s}\end{array}$ & $\begin{array}{c}\text { qmax. } \\
\text { pour B.V. } \\
\text { en } \mathrm{m}^{3} / \mathrm{s} / \mathrm{km}^{2}\end{array}$ & $\begin{array}{c}\text { Qmax. } \\
\text { pour B.V. } \\
\text { total en } \mathrm{m}^{3} / \mathrm{s}\end{array}$ \\
\hline ASO & $\begin{array}{c}3,5 \rightarrow \\
18,5 \rightarrow\end{array}$ & $\begin{array}{c}30 \text { pour } 8,5 \mathrm{~km}^{2} \text { à } P 2 \\
\text { totar } 2 \mathrm{~km}^{2} \text { aval }\end{array}$ & $\begin{array}{c}3,5 \text { à } 6,4 \\
\text { pour } 10,5 \mathrm{~km}^{2}\end{array}$ & 37 à 67 \\
\hline BETES & 18,7 & 60 pour $3,2 \mathrm{~km}^{2}$ à $P 6$ & $\begin{array}{c}18,7 \text { pour } \\
4,38 \mathrm{~km}^{2}\end{array}$ & 82 \\
\hline SELVA & 18,6 & 40 pour $2,15 \mathrm{~km}^{2}$ à $P 9$ & 18,6 pour $2,75 \mathrm{~km}^{2}$ & 51,5 \\
\hline ARAS & 9,65 à 11,3 & $\begin{array}{c}\text { Aso }+ \text { Betes }+ \text { Selva } \\
170 \text { à } 200 \text { pour } 17,6 \mathrm{~km}^{2}\end{array}$ & $\begin{array}{c}9 \text { à } 11 \\
\text { pour } 18,8 \mathrm{~km}^{2}\end{array}$ & $180-210$ \\
\hline
\end{tabular}

\subsection{Débit de pointe à l'exutoire du rio Aras}

On obtient les débits à l'exutoire de l'Aras par simple sommation des débits de chacun des affluents, compte tenu que le Selva, le Betes et la zone terminale de l'Aso ont des temps de concentration proches, compris entre $1 / 2$ heure et $3 / 4$ d'heure. On extrapole finalement pour l'Aras, par proportionnalité de surface, un débit de pointe compris entre 180 et $210 \mathrm{~m}^{3} / \mathrm{s}$ en ordre de grandeur, soit des débits spécifiques compris entre 9 et $11 \mathrm{~m}^{3} / \mathrm{s} / \mathrm{km}^{2}$ pour $18,8 \mathrm{~km}^{2}$ de bassin versant.

\subsection{Durée de retour de la crue liquide}

L'estimation de la fréquence d'une telle crue doit être abordée avec circonspection du fait de la méconnaissance de l'hydrologie du bassin versant et de l'absence de données pluviographiques. La méthode du Gradex est appliquée sur les maxima annuels des pluies de $24 \mathrm{~h}$ de la station de Biescas (tabl. I) avec les hypothèses suivantes :

- un débit moyen journalier de fréquence $1 / 10$ de $20 \mathrm{~m}^{3} / \mathrm{s}$ comme point pivot, par analogie avec différents torrents des Pyrénées nord-occidentales,

- le coefficient $R$ (débit de pointe/débit moyen journalier) est pris égal à 2 .

Les ratios entre les débits estimés de la crue et le débit décamillénal, $110 \mathrm{~m}^{3} / \mathrm{s}$ (tabl. 4 ), sont de 1,66 et 1,94 . Ils se situent dans l'intervalle des ratios, débits de la crue maximale probable (PMF)/crue décamillennale (méthode du Gradex), reconnus pour des bassins montagneux ou à forts effets orographiques [4]. Ces éléments confèrent à la crue du 7 août 1996 un caractère exceptionnel en fréquence et situent l'ordre de grandeur de la crue maximale probable sur cette région morphoclimatique.

Tableau 4. - Débits de pointe de fréquences rares du rio Aras par la méthode du Gradex.

\begin{tabular}{|c|c|c|c|}
\hline Fréquence & $1 / 100$ & $1 / 1000$ & $1 / 10000$ \\
\hline $\mathrm{O} /\left(\mathrm{m}^{3} / \mathrm{s}\right)$ & 63 & 85 & 108 \\
\hline
\end{tabular}

\subsection{Discussion}

Cette estimation des débits liquides diffère sensiblement des résultats obtenus par une équipe espagnole [5]. Le débit de pointe est estimé à $500 \mathrm{~m}^{3} / \mathrm{s}$ pour le rio Aras sur des sections d'écoulement reconstituées au droit des barrages. Cette estimation utilise une formule de déversoir, ce qui est peu fondé car les vitesses d'approche sur les ouvrages étaient élevées. De plus, la forte végétalisation initiale des plages de dépôts, l'importance du transport solide et l'ampleur des affouillements, sont autant d'éléments qui nous ont conduit à considérer ces sites comme peu fiables pour la reconstitution des débits. A la suite, le débit de $300 \mathrm{~m}^{3} / \mathrm{s}$ estimé pour le rio Betes résulte de mesures réalisées dans le bief terminal du Betes profondément érodé par des écoulements à forte concentration solide. Il en découle une surestimation des débits réels, telle que nous l'avons évoquée pour les sections P3-P4-P5 sur l'Aso.

\section{L'ÉCOULEMENT DE LA CRUE DANS LE CHENAL DE L'ARAS}

Le chenal de l'Aras a fait l'objet entre 1940 et 1950 d'une correction torrentielle de grande ampleur. L'objectif principal était de fixer le profil en long par la construction d'une quinzaine de seuils et barrages en pierres maçonnées. Les ouvrages les plus importants dépassent 10 mètres de haut et 30 à 35 mètres de long. Il s'agit donc d'ouvrages considérables compte-tenu de la technique de mise en ouvre.

Le 7 août 1996, les retenues étaient comblées. Sur les coupes ouvertes dans les cuvettes partiellement vidangées de leur contenu détritique durant la crue, on observe un dispositif stratigraphique de couches sub-horizontales présentant divers faciès : argiles de décantation reliées à la mise en eau temporaire de la retenue; dépôts hétérométriques de crues torrentielles à forte charge solide, pour l'essentiel (photo $\left.n^{\circ} 4\right)$

La rupture de la quasi-totalité de ces ouvrages a libéré tout ou partie de leur contenu détritique. La sédimentation d'environ $70000 \mathrm{~m}^{3}$ de matériaux sur la moitié droite du cône de déjection associée à un écoulement en masse de type granulaire constituent l'autre fait majeur de la crue de l'Aras.

\subsection{Modalités de la rupture des seuils}

On a évoqué le rôle positif des seuils disposés aux exutoires de l'Aso et du Selva qui ont provoqué la sédimentation des coulée de débris et atténué les reprises érosives. Sur le cours terminal du Betes à très forte pente, les deux ouvrages ont été balayés par la crue.

Dans le chenal de l'Aras, le dispositif de correction torrentielle a été largement débordé. Le premier barrage a cédé du fait de l'action des écoulements liquides issus du Betes, soit $80 \mathrm{~m}^{3} / \mathrm{s}$ en pointe.

Sur le premier ouvrage, la rupture se limite à une brèche alignée dans l'axe du rio Betes qui a incisé un chenal dans le remplissage sédimentaire. Ce chenal suggère que la rupture résulte du creusement d'une fosse à l'amont du seuil indui- 


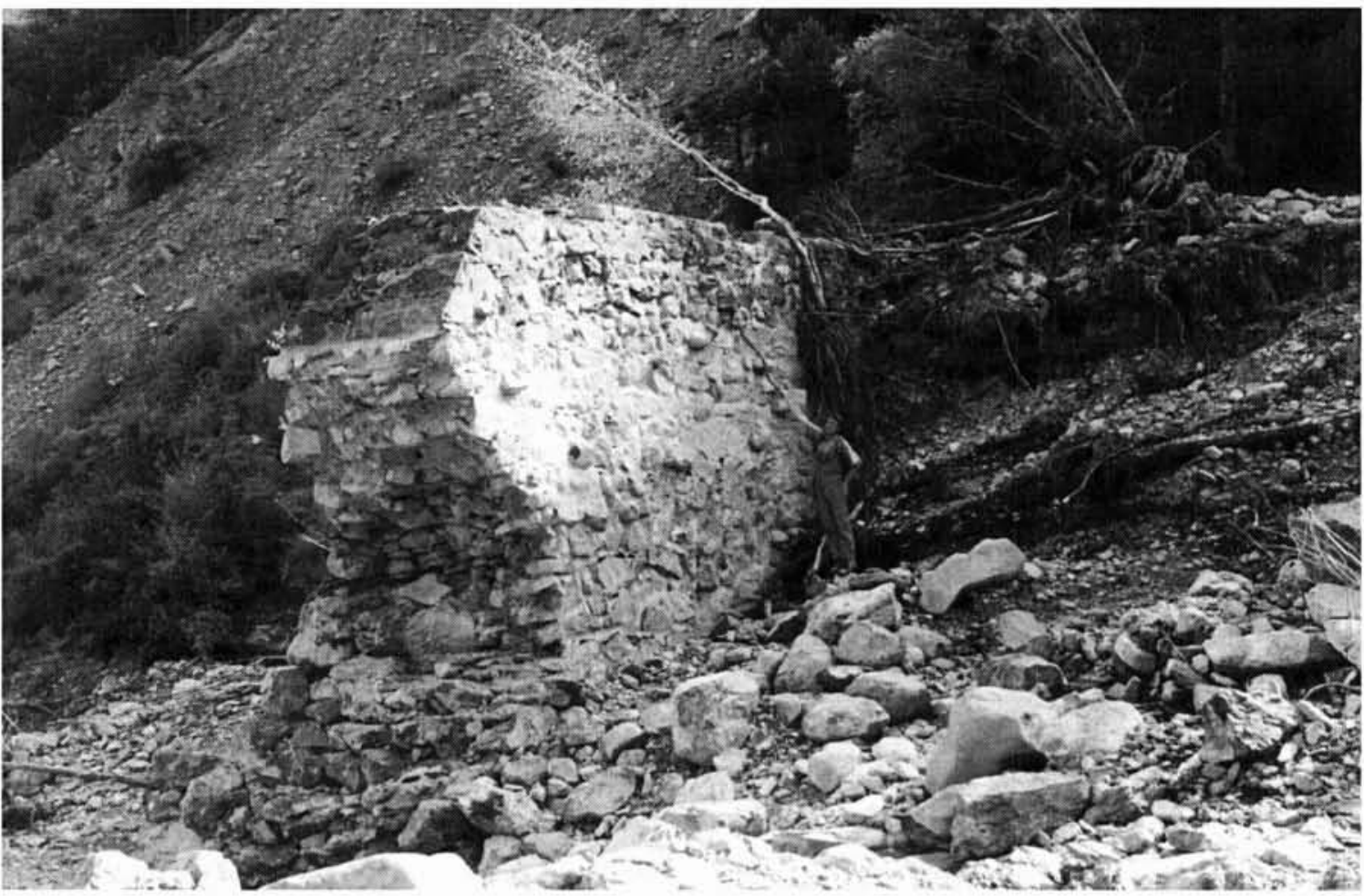

Photo $\mathrm{n}^{\circ}$ 4. $2^{\mathrm{e}}$ seuil sur le rio Aras vidangé de son contenu sédimentaire du fait d'une rupture massive ; mur en pierres et mortier sec.

sant un enfoncement régressif de l'écoulement dans les dépôts de la retenue [3]. Une rupture par affouillement du pied de l'ouvrage aurait probablement conduit à une rupture plus massive de la digue. Dans l'axe de l'Aso, l'ouvrage n'a pas été endommagé.

Dès le second seuil qui concentre les eaux des trois affluents, le débit liquide en pointe, entre 170 et $200 \mathrm{~m}^{3} / \mathrm{s}$ induit très clairement un degré de destruction bien supérieur: il ne reste ici qu'un pan de mur en rive droite (photo $\left.\mathrm{n}^{\circ} 4\right)$.

Sur les autres seuils, l'ampleur des désordres rend difficile l'analyse des mécanismes de rupture. On observe que le $4^{\mathrm{e}}$ barrage a cédé latéralement du fait d'un glissement dans le versant morainique probablement initié par le contournement de l'ouvrage. Les quelques édifices qui ont résisté, le doivent à la présence de blocs cyclopéens (plus de $10 \mathrm{~m}$ de hauteur) sur lesquels ils ont été fondés.

\subsection{Constitution de la coulée de débris « terminale »}

A l'aval du premier seuil, les ruptures d'ouvrages se sont succédées évoquant une sorte de réaction en chaîne libérant une grande partie des sédiments stockés.

On vient de voir le rôle déterminant des écoulements liquides dans la rupture des ouvrages. Selon le schéma dynamique à la pointe de la crue, la phase d'écoulement liquide fortement érosive succède à une phase de sédimentation identifiée précédemment aux bas des affluents et en particulier sur le Betes. Au-delà, on ne dispose pas d'éléments permettant d'identifier sûrement la dynamique de la crue. Néanmoins, le scénario suivant est envisageable.

La transformation en écoulement en masse (phase 2) est rapide et la coulée de débris sédimente rapidement (phase 3 ) après franchissement d'un ou deux seuils. La reprise érosive immédiate (phase 1) déstabilise le seuil ayant bloqué la coulée et mobilise un volume sédimentaire accru qui évolue rapidement en une nouvelle coulée de débris (phase 2). Cette séquence a pu se répéter jusqu'à ce que l'écoulement en masse acquière le volume et la hauteur lui permettant de s'affranchir des pertes de charge imposées par les ultimes seuils.

La coulée "terminale " a évolué sur une pente moyenne de $15 \%$ puis s'est étalée en éventail sur la moitié droite du cône de déjection. Le front de la coulée s'est bloqué à la limite amont du camping (fig. 3 ), ce qui permet de dire que la catastrophe aurait pu être pire si la coulée de débris s'était avancée de quelques dizaines de mètres du fait d'un volume supérieur. Ce volume "manquant " se situe notamment au niveau des 4 ou 5 seuils qui ont résisté à la crue.

\subsection{Modalités de l'écoulement en masse}

Les caractéristiques sédimentologiques des dépôts d'écoulements en masse contenus dans le Betes, le cours aval de l'Aso et plus accessoirement sur le Selva nous conduisent à évoquer un mode d'écoulement de type granulaire. La texture de la matrice, faiblement argileuse, est sablo-silteuse; la structure du mélange est toujours à support granulaire (« clast-support », c'est-à-dire que les grains grossiers présentent des contacts longs et ne «flottent " pas dans la matrice, largement minoritaire en volume; les marques d'impact sur les grains montrent l'importance des interactions granulaires; la concentration des plus gros éléments au toit du dépôt suggère une ségrégation verticale et l'existence d'un gradient granulométrique inverse. Ces faits caractérisent bien un dépôt d'écoulement en masse granulaire $[5,6,7]$.

Les dépôts étalés sur le cône de déjection présentent les mêmes traits sédimentologiques auxquels s'ajoutent une 
structuration particulière, peu évoquée dans la littérature. On observe en effet un gradient granulométrique longitudinal inverse, soit une croissance granulométrique amont-aval. Cela a été notamment observé par Mears [8] et sur des corps bien individualisés d'extension décamétrique dans les Pyrénées nord-occidentales. Nous n'avons pas pu mesurer cette évolution granulométrique longitudinale du fait de l'interdiction de pénétrer sur le périmètre du camping. Nous avons pu simplement constater l'absence de blocs supra-métriques dans la partie amont du dépôt, ces blocs devenant progressivement dominants vers l'aval. Ce dispositif ne peut pas être confondu avec la structuration longitudinale d'une coulée de débris boueuse caractérisée par la présence d'un front granulaire, plus grossier que le reste de la coulée. Cette structuration pourrait aussi résulter de la superposition de coulées de débris de granulométries différentes, mais la morphologie du dépôt montre une homogénéité du corps sédimentaire ne suggérant pas une mise en place polyphasée. Enfin, la sédimentation de dépôts charriés en fin de crue visibles sur la partie amont de la coulée n'a modifié que partiellement la granulométrie initiale et n'explique pas l'absence de gros blocs.

\section{CONCLUSION}

La reconstitution de la crue du 7 août 1996 du torrent Aras est riche d'enseignements de portée générale quant à la "compréhension" de la dynamique d'une crue torrentielle majeure et à la définition des stratégies de prévention et de protection, ainsi que dans le cadre régional des Pyrénées occidentales.

\subsection{Les enseignements généraux de la crue}

En premier lieu, la catastrophe humaine est induite par un écoulement liquide considérable (sans considérer les questions de localisation et d'autorisation du camping qui sont in fine déterminantes) alors que le problème des transports solides constitue, à juste titre, la préoccupation dominante en matière de prévention des crues torrentielles. Les barrages de sédimentation permettent de réduire, voire d'annihiler cet aspect de l'aléa torrentiel. La crue de Biescas montre qu'il demeure considérable dans le cas de pluies extrêmes. Il est donc de première importance d'intégrer le risque d'une crue liquide extrême dans les études de définition des risques torrentiels, notamment pour l'établissement des Plans de Prévention des Risques.

Les ouvrages de correction torrentielle ont eu un comportement différencié en fonction de la valeur des débits liquides. Ces ouvrages en pierres ont été déstabilisés dès que les écoulements liquides ont pu affouiller en amont ou en pied. Il est cependant difficile d'estimer un seuil de comportement des ouvrages sur la seule valeur des débits, d'autres paramètres devant être pris en compte. Quoiqu'il en soit, ces ouvrages pionniers de la correction torrentielle ont montré leurs limites dans le cadre d'une crue liquide extrême. Cela devrait inciter à une vigilance accrue sur les ouvrages analogues et, dans certains cas, à programmer leur modernisation progressive. Mais les ouvrages modernes ont aussi leur limite. L'instabilisation latérale de certains seuils sur des terrains faiblement consolidés impose des ancrages très profonds, tant verticalement que latéralement (rupture partielle du barrage amont du rio Selva).

Enfin, la crue du torrent Aras montre que le premier élément de la protection contre les crues (torrentielles) est la non-exposition des personnes et des biens. La catastrophe humaine ne résulte ainsi que de la seule présence du camping sur un cône de déjection actif. Les aménagements de correction torrentielle n'avaient pas été conçus pour permettre l'implantation d'un camping mais, il y a quelque 50 ans, pour tempérer les crues torrentielles du point de vue des transports solides et des divagations sur le cône de déjection. Ces éléments (correction torrentielle et protection du camping) étaient donc historiquement et techniquement disjoints.

\subsection{Pluie et crue maximale probable dans les Pyrénées occidentales}

Nous avons souligné que les Pyrénées occidentales se caractérisent par l'occurrence de phénomènes pluvieux extrêmes. $\mathrm{Si}$ les versants nord et sud des Pyrénées montrent, du fait de la disposition est-ouest de la chaine, des différences climatiques marquées, il est probable que les phénomènes pluvieux "plafonds " soient fortement analogues au nord comme au sud si l'on considère les relevés pluviométriques récents.

Aussi, la pluie et la crue du 7 août 1996 doivent-elles constituer une référence en matière de prévention des risques torrentiels dans les Pyrénées-occidentales. Dans ce sens, la crue de Biescas constitue un repère de la crue torrentielle maximale dans cette région qui doit servir de base de réflexion et d'action en matière d'aménagement de l'espace montagnard.

\section{RÉFÉRENCES}

[1] Lajournade C., \& Lalanne-Berdoutice G. (1995). «Contextes pluviométrique, géomorphologique et anthropique des crues extrêmes dans les Pyrénées occidentales». Actes du colloque «Crues, bassins versants et lits fluviaux ». C.N.R.S., Paris. Publié dans Géomorphologie, 1997, nं 2, 111-120.

[2] Koulinsky V. (1993). - « Etude de la formation d'un lit torrentiel par confrontation d'essais sur modèle réduit et d'observations de terrain ». Thèse de l'Université de Grenoble 1, collection « Etudes du CEMAGREF » série Equipements pour l'eau et l'environnement, n¹5, 538 p., 1994.

[3] Couvert B., Lefebvre, Lefort \& Morin. (1991). « Etude générale sur les seuils de correction torrentielle et les plages de dépôts ». La Houille Blanche, 6, 449-456.

[4] Collectif (1994), - « Les crues de projet des barrages: méthode du GRADEX ». $18^{e}$ Congrès CIGB/ICOLD. Barrages et Réservoirs $n^{\circ} 2$.

[5] White Sue, Garcia Ruiz J.M. , Marti C., Valero C., Paz Errea M. \& Gomez Villar A.. (1997). - « The 1996 Biescas Campsite disaster in the central spanish Pyrenees and its temporal and spatial context ». Hydrological processes, 11, 1797-1812.

[6] LowE D.R. (1976). - « Grain flow and grain flow deposits. Journal of sedimentary Petrology, 46, 1, 188-199.

[7] LowE D.R. (1979). — « Sediment gravity flows : their classification and some problems of application to natural flow and deposits ". Journal of sedimentary Petrology, 27, 75-82.

[8] Nemec W. \& Steel R.J. (1984), - «Alluvial and coastal conglomerates : their significant features and some comments on mass-flow deposits ». In : Koster-Steel (Eds), « Sedimentology of gravels and conglomerates "; Canadian Society of Petroleum Geologists, memoir 10, 1-31.

[9] Mears A.I. (1979). - «Flooding and sediment transport in a small alpine drainage basin in Colorado ». Geology, 7, 53-57. 